\title{
A Mixed-Integer Linear Programming Model for Optimizing the Scheduling and Assignment of Tank Farm Operations
}

\author{
Sebastian Terrazas-Moreno ${ }^{\mathrm{a}}$, Ignacio E. Grossmann ${ }^{\mathrm{a}^{*}}$, John M. Wassick ${ }^{\mathrm{b}}$ \\ ${ }^{a}$ Carnegie Mellon University, 5000 Forbes Ave, Pittsburgh PA 15232, USA \\ ${ }^{b}$ The Dow Chemical Company, 1776 Building, Midland, MI 48674, USA
}

\begin{abstract}
This paper presents a novel mixed-integer linear programming (MILP) formulation for the Tank Farm Operation Problem (TFOP), which involves simultaneous scheduling of continuous multi-product processing lines and the assignment of dedicated storage tanks to finished products. These products are not allowed to mix in storage tanks. Therefore, once an assignment is made, it has to be maintained until the end of the operating horizon. Since all products processed by finishing lines have to go into the tank farm before being shipped, there is the potential to run out of storage, ultimately impacting the throughput of the finishing lines, a condition known as blocking. The objective of the problem is to minimize blocking of the finished lines by obtaining an optimal schedule and an optimal allocation of storage resources. The scheduling part of the model is based on the Multi-operation Sequencing (MOS) model by Mouret et al., (2011). The formulation is tested in three examples of different size and complexity. The possibility of incorporating the MILP model into a decision support system in combination a Discrete Event Simulation (DES) model of a tank farm is also discussed.
\end{abstract}

Keywords: Tank farm operation, production scheduling, tank assignment, multioperation sequencing.

\section{Introduction}

Chemical manufacturing sites ship finished products to customers using different modes of transportation (MOT) such as railcars, tank trucks, and pipelines. These MOTs are usually loaded from or connected to storage tanks. Consequently, all finished products have to be fed from the process into the storage tanks before being shipped to customers. This type of operation imposes the need for available storage space at all times in order to 
avoid unnecessary shut-downs of the upstream chemical process. When these shutdowns occur, they are said to be a result of storage tanks blocking the process. If the chemical process produces several products and each one requires dedicated tanks, the producttank assignment and the processing schedule at the finishing lines determines how efficiently the storage space is used. An inefficient assignment of tanks or processing schedule can result in blocking the production of certain products, even when there is plenty of available storage space in tanks assigned to other products.

In this paper, we consider a multiproduct manufacturing facility that includes finishing lines connected to a number of storage tanks from which products are shipped to customers using different modes of transportation (MOT). The tanks are dedicated to one product, meaning that once a product is assigned to a tank, it cannot be used for another product. Cleaning of tanks to store different products is not allowed. We are concerned with finding the best possible assignment of products to dedicated storage tanks, and the best processing schedule at the finishing lines in order to minimize the unallocated production. For scheduling purposes, there is a set of production orders that requires the processing of a certain amount of product in the finishing lines. Orders have a known release date and all are due at the end of the time horizon. The release date corresponds to the moment when a certain amount of an unfinished product becomes available for processing in the finishing lines. These lines can process the order immediately and feed the storage tanks, or delay the order for a while until there is available storage space. The main decisions in this problem are the tank-product assignment and the scheduling of processing orders. The set of storage tanks included in the production facility is collectively known as a Tank Farm, and therefore, the problem outlined above is referred to in this paper as the Tank Farm Operation Problem (TFOP).

The paper by Sharda and Vazquez (2009) illustrates the relevance of the tank farm operation problem (TFOP) for the Dow Chemical company. The authors describe the development of a Decision Support System to evaluate the operation of a tank farm at a chemical production site in Freeport, TX. The system they propose is based on Discrete Event Simulation (DES). In discrete event models the system changes states as events 
occur and only when those events occur (ExtendSim, 2007). DES works by representing the occurrence of an event by generating and passing items among the elements of the simulation model. Cassandras et al. (1993), Banks et al. (2005), and ExtendSim (2007) are useful references on Discrete Event Systems and Discrete Event Simulation. Sharda and Vazquez (2009) evaluated the operation of a tank farm in a Dow Chemical site that consists of more than 80 tanks for storing 60 product families, processed in 6 finishing lines. Their simulation model captures complex operating constraints, such as the effect of recycle lines on the simultaneous loading and unloading of tanks, operational logic when dealing with delayed processing orders, and the stochastic nature and dynamics of the operation of loading from tanks unto the different modes of transportation (MOT). Since the approach is based on simulation, it is not intended for finding an optimal resource allocation or production schedule; it is a tool for evaluating different storage tank allocations for a given production schedule.

Other authors have used this type of DES as a support tool for operating a tank farm. Chen et al. (2002) from the Mathematical Modeling Group at BASF provide another example of a DES study of logistics in a chemical plant. The tank farm problem is also relevant in crude refining operations. For instance, Stewart and Trierwiler (2005) carried out a study of the tankage requirement in different operational scenarios for the Kuwait National Petroleum Company. Their main tool is DES which they combine with Linear Programming (LP). Chryssolouris et al. (2005) present an integrated simulation based approach that manages scheduling, tank farm, inventory, and distillation operations in a refinery. Their approach is based on generating random solutions within a given search space and evaluating them using the simulation model.

Mathematical programming is another approach to the Tank Farm Operation Problem (TFOP). Optimization methods can determine the best possible tank allocation and/or production schedule within a given search space, as opposed to simulation tools that require the allocation and schedule to be specified. The disadvantage of this approach is that it requires operational constraints to be expressed as algebraic equations. Many of the operational constraints of TFOP have to be simplified in order to express them with 
algebraic equations for the optimization algorithms. Even with this limitation, optimization approaches have been successfully used in problems related to storage tank allocation and tank transfer scheduling. Hvattum et al. (2009) addressed the storage tank allocation problem (TAP) in a maritime bulk shipping operation. A ship is equipped with a set of storage tanks that are loaded and unloaded according to a scheduled route. This problem has similar constraints as the tank farm operation problem we address in this paper; each tank holds only one type of product and several tank-product assignments are infeasible due to structural or safety constraints. The loads received and delivered at different ports are similar to product loading and unloading to storage vessels in the tank farm according to a production and shipping schedule. A Mixed-integer Linear Programming (MILP) model is used to either test the feasibility of a shipping route, to minimize tank cleanup time, or to maximize the vacant space in storage tanks. The main difference between the TAP and the TFOP is that the TFOP deals with a continuous production system, whereas the TAP deals with a limited number of discrete loading and unloading events. The work by Ha et al. (2000) is a good example of a research topic related with storage tank allocation, namely, the optimization of intermediate buffer sizing and allocation in multi-product batch process systems. Ha et al. (2000) determine the location, number, and size of storage tanks with the objective of minimizing the makespan. As opposed to the Tank Farm Operation Problem (TFOP), storage vessels can be shared by different types of batches. Vecchietti and Montagna (1998) address a similar problem.

From the above review, we can conclude that Discrete Event Simulation (DES) is the approach that has received the most attention for addressing the TFOP in process industries. Mathematical optimization has been mostly aimed at related problems such as buffer tank allocation in multiproduct batch scheduling, or to assignment problems such as in the case of the tank allocation problem in maritime operations. Some authors (Zeng and Yang, 2009; Chen et al., 2002) have correctly pointed out that the number of variables and constraints, the operational complexity, and the stochastic nature of logistic processes involved in tank farm management produce either intractable or oversimplified mathematical optimization models. Zeng and Yang (2009) proposed integrating 
simulation and optimization for solving the TFOP. Their argument is that an approximate optimization model is sufficient for obtaining good solutions that can be evaluated using the simulation model in a second step. They used neural networks and genetic algorithms for the optimization module.

The objective of this paper is to present an optimization method based on mathematical programming, namely Mixed-Integer Programming (MILP), for solving the TFOP. Our model of the TFOP includes scheduling of orders that arrive to the finishing lines as well as the optimal tank allocation. We use the description of the tank farm operation given by Sharda and Vazquez (2009), and try to incorporate in our MILP model as much of the operational details as possible. This paper does not include simulation by DES. Nevertheless, we believe that the output of the optimization model we propose could be evaluated, validated, and communicated using DES.

In the following sections of this paper we give a more detailed description of the tank farm problem, state the optimization problem, discuss the mathematical model, and apply the formulation to three case studies. We end this paper with a summary of our findings and a discussion of possible future work.

\section{Problem Statement}

A downstream section of a chemical production facility includes a set $M$ of continuous finishing lines that represent the last step in the manufacturing of a set $J$ of products, along with a set $K$ of storage tanks (the tank farm) from where the products are shipped to the consumers. The shipping operation uses different modes of transportation, usually railcars, tank trucks, or pipelines. Figure 1 (Sharda and Vazquez, 2009) shows a representation of this system. 


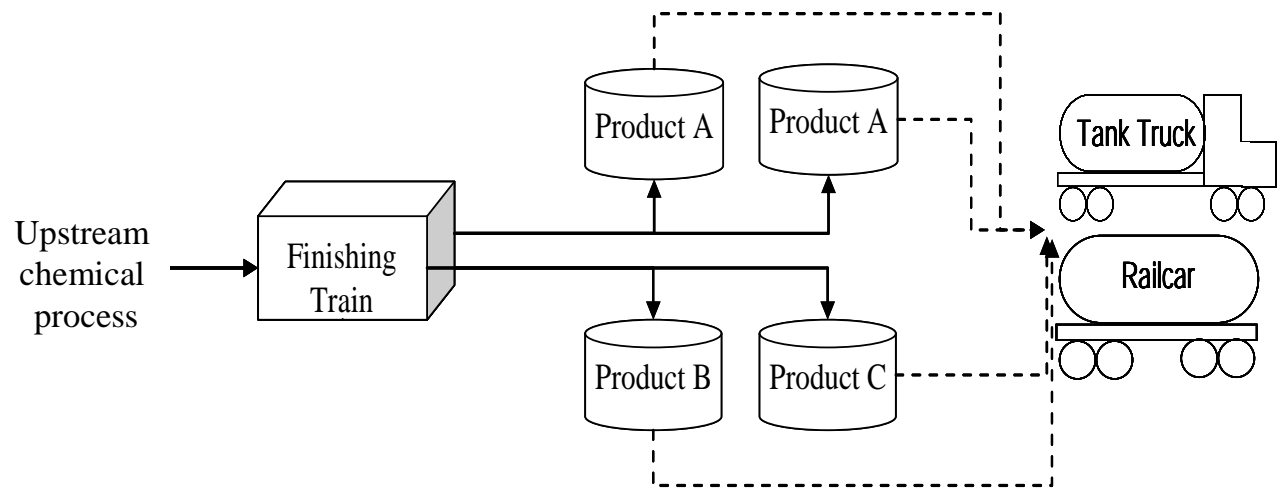

Fig. 1 Downstream section of chemical production process

The processing step in lines $m \in M$ is carried out according to processing orders. A processing order is generated when a batch of unfinished product from the upstream chemical plant is ready to be processed by the finishing lines, which operate continuously. The parameter $d_{o, j}$ specifies the amount of product $j$ to be produced according to order $o$. Each order $o \in O$ has a release date $r d_{o}$ that corresponds to the moment when the unfinished product is available for processing in the finishing lines, and when the corresponding production order gets generated. All orders are due at the end of the operating horizon. If an order cannot be processed at its release date, its production can be delayed as long as it can still meet the due date. The production rate of each product $j \in J$ in line $m$, rate $_{j, m}$, is a known deterministic parameter. After its release date, $r d_{o}$, the order $o$ is processed in the finishing lines and sent to storage in one of the $k \in K$ tanks of the tank farm. At the beginning of the time horizon, some or all of the tanks are empty. However, once a tank $k$ has been allocated to store a product $j$, it remains dedicated to this product throughout the operating horizon considered in this paper. One of the biggest operational challenges of this problem is that an order cannot be processed and transferred to a tank, unless there is available space in the tanks. When transfer is not possible and the production order has to be delayed, it is said that tanks are 
blocking the finishing lines. In this case the processing of orders in the finishing lines has to be rescheduled.

Figure 2 is adapted from Sharda and Vazquez (2009); it shows the operational logic for processing production orders and transferring finished product into storage tanks.

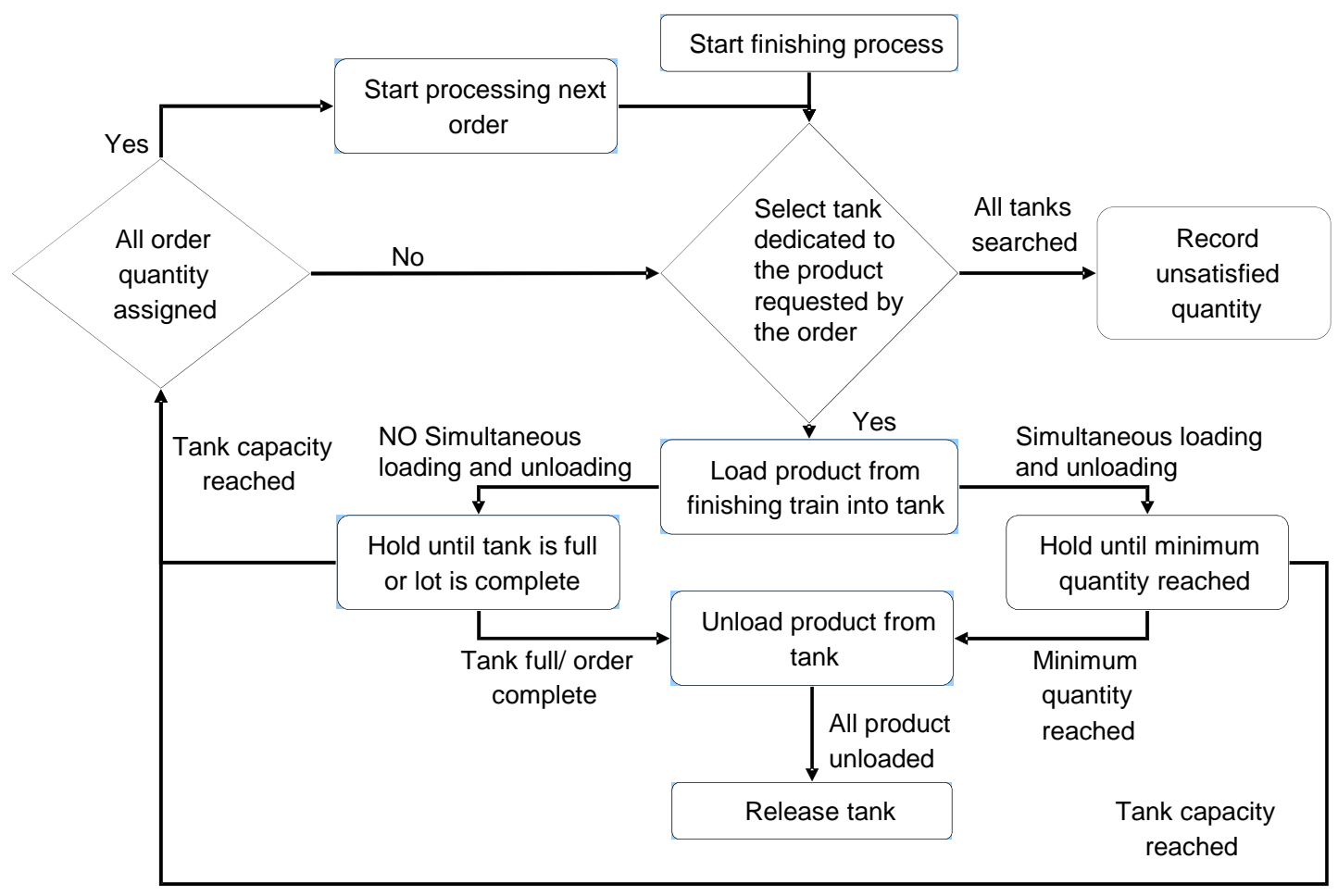

Fig. 2 Operational logic in tank farm simulation model

Since the time horizon is finite, blocking and delaying or orders ultimately results in some orders that cannot be produced. Such unsatisfied orders generate unallocated product at the end of the time horizon. The main decisions that this paper is concerned with are the assignment of finished products to storage tanks, and the scheduling of production orders.

There are a number of operational characteristics that make the management of an actual tank farm a complex problem. The processing of certain products might not be possible in every finishing line, and a product can only be transferred from a line to a tank if there is piping or some other type of connection between them. Simultaneous loading and unloading is prohibited for some tanks. Finally, shipping can be done using different 
modes of transportation (MOTs), namely, railcars and tank trucks that are loaded in batches at specified times that correspond to the arrival of available MOTs.

The problem can be summarized as follows:

Given are:

- A finite time horizon

- A set of finishing lines and their operational characteristics:

o Subset of products that can be processed in it

o Processing rate for each product

o Subset of tanks that the line is connected to, and to which it can transfer material

- A set of production orders with the following information:

o Product to be processed

o Quantity of product to be processed

o Release date

o A due date corresponding to the end of the operating horizon

- A set of products

- A set of storage tanks and their characteristics:

o Maximum capacity (volume or mass)

o Subset of products that are compatible with its operating conditions

o Frequency of unloading to MOT

o Rate of transfer to MOT during unloading

The simplifications with respect to realistic tank farm operation are as follows:

- Unloading operations occur at the same time in all tanks. Otherwise, the unloading of each tank at the end of each day could potentially require a priority slot (defined later in the paper), and the problem size would increase considerably.

- Simultaneous loading and unloading of tanks is prohibited. 
- Modes of transportation operate in semi continuous mode only; there are no continuous MOTs.

- Changeover times are neglected for the scheduling.

The problem is to determine:

- The assignment of products to dedicated storage tanks

- The assignment of orders to finishing lines

- The start and duration of the processing time for each order

- The tank or tanks to which each order will be transferred to

\section{Subject to:}

- Assignment constraints:

o One product per tank

o Maximum and minimum number of tanks for a product

o Physical and chemical compatibility of finished product and tank conditions

o Constraints given by existing connections between finishing lines and storage tanks

- Mass balance constraints

o The rate of processing of an order is equal to the rate of transfer to storage tanks

o Accumulation in storage tanks is equal to all transfers from lines minus all quantity loaded to MOTs

- Scheduling constraints

o No two orders can be processed simultaneously in the same line

o An order can only be processed after its release date

- Tank operation constraints

o Simultaneous loading and unloading is not allowed for some tanks

o Unloading operations to MOTs occur with predetermined frequency

o Unloading operations to MOTs have a predetermined maximum duration 
With the objective of:

- Minimizing unallocated production that results from blocking finishing lines by unavailability of storage space

\section{Continuous time scheduling in the Tank Farm Operation Problem (TFOP)}

The fact that production orders can be delayed, allows for optimization of the processing schedule at the continuous finishing lines. An important characteristic of the finishing process is that once an order has started, it has to be processed completely or the unprocessed quantity has to be declared as unallocated product. This fact is a result of the operational logic shown in Figure 2.

There are alternative models that can be used for scheduling continuous parallel production lines. Since we are interested in the material balance in the storage tanks, we need to consider the transfer of different products from several parallel finishing lines. The simplest alternative to do so is to use a discrete time formulation. The state-tasknetwork (Kondili et al., 1993) and the resource-task-network (Pantelides, 1994) are the most general discrete time formulations for batch processes. Since the process at hand is single-stage and continuous, a much simpler formulation where at most one production order can be assigned to each time interval and each line could be used. The transfer to storage tanks would be equal to the processing rate, and the unloading of tanks to MOTs could also be specified for some of the time intervals. The balance of the inventory tank could be easily calculated at the end of each time interval. The drawback of this simple discrete time formulation is that when a large number of time intervals are needed, the problem may become intractable.

Continuous time scheduling models are now common since they can potentially decrease the combinatorial complexity that results from discrete time models (Floudas and Lin, 2004). Erdirik-Dogan and Grossmann (2008) present a model for simultaneous planning and scheduling of continuous parallel production lines that divides the operating horizon into planning time periods. The inventory mass balance is calculated at the beginning and 
at the end of each time period. A slot-based MILP scheduling problem in continuous time is solved within each time period. This model has a very natural way for incorporating sequence-dependent changeovers that has been extended by Lima et al. (2011) and Kopanos et al. (2011) to consider production changeovers across time periods. Yet another type of continuous time models relevant to the TFOP comes from the study of tank transfer and crude oil scheduling problems in the refining industry (Furman et al., 2007; Mouret et al., 2009; Mouret et al., 2011 ). Among these alternative models, we use the Multi-operation Sequencing (MOS) model described by Mouret et. al (2011). In Appendix A we discuss how the MOS model was chosen over some alternative formulations.

\section{Mathematical Model}

The mathematical model in this paper is based on the Multi-operation sequencing (MOS) model of Mouret et. al (2011). It has been modified to account for specific considerations of the TFOP. The MOS formulation by Mouret et. al (2011) uses the idea of operations assigned to priority time slots that enforce a precedence of time events. Multi-operation sequencing (MOS) receives its name from the fact that several operations can be assigned to the same slot unless they are considered as non-overlapping. To illustrate this concept, consider the six operations and three resources shown in Table 1 (Mouret et al., 2011). Operation $v_{4}$ consumes resources $r_{1}$ and $r_{2}$, which means it cannot be performed simultaneously with either operation $v_{1}$ or $v_{2}$. Operations that share resources are termed non-overlapping.

Table 1. Set of operations and resources used by each operation

\begin{tabular}{llllccc}
\hline Operation & $v_{1}$ & $v_{2}$ & $v_{3}$ & $v_{4}$ & $v_{5}$ & $v_{6}$ \\
Resource & $r_{1}$ & $r_{2}$ & $r_{3}$ & $r_{1} \wedge r_{2}$ & $r_{1} \wedge r_{3}$ & $r_{2} \wedge r_{3}$ \\
\hline
\end{tabular}

Figure 3 (Mouret et al., 2011) shows another way of representing operations and the nonoverlapping relationship among some of them. The graph in this figure has an arc between all non-overlapping operations. The idea of a clique from graph theory is used to group subsets of non-overlapping operations. For instance, the subset $\left\{v_{2}, v_{4}, v_{6}\right\}$ is a 
clique in the graph on Figure 3. Figure 4 (Mouret et al., 2011) shows a schedule with the six operations in Table 1 that illustrates the idea of multi-operation sequencing (MOS) and non-overlapping operations for a set of 4 priority time slots. For instance, note that operations $v_{1}$ and $v_{6}$ are assigned to slot 1 , while operations $v_{2}$ and $v_{5}$ are assigned to slot 2. In general, let $V$ be the set of operations, $L$ the set of slots, $S_{v, \ell}$ the start of operation $v \in V$ in slot $\ell \in L, E_{v, \ell}$ the ending time, and $Z_{v, \ell}$ a binary equal to 1 if $v$ is assigned to $\ell$. Then, the basic idea of the MOS model is summarized by the following constraints:

$$
\sum_{v \in V^{*}} Z_{v, \ell} \leq 1 \quad \ell \in L, V^{*} \in \operatorname{clique}(V)
$$

and

$$
\sum_{v \in V^{*}} E_{v, \ell_{1}} \leq \sum_{v \in V^{*}} S_{v, \ell_{2}}+H\left(1-\sum_{v \in V^{*}} Z_{v, \ell_{2}}\right) \quad \ell_{1}, \ell_{2}, \in L, \ell_{1}<\ell_{2}, V^{*} \in \operatorname{clique}(V),
$$

where $\operatorname{clique}(V)$ is formed by all subset of operations $V^{*} \subseteq V$ such that any two operation in $V^{*}$ must not overlap. For instance, all operations that use a common resource would be part of a set $V^{*} \in \operatorname{clique}(V)$. Note that constraints (MOS1) states that at most one operation $v$ from a given subset of operations $V^{*}$ can be assigned to a slot $\ell$ , while constraint (MOS2) enforces for all operations from the subset $V^{*}$, that the end time of slot $\ell_{1}$ takes place before the start time of slot $\ell_{2}$ if an assignment is made.

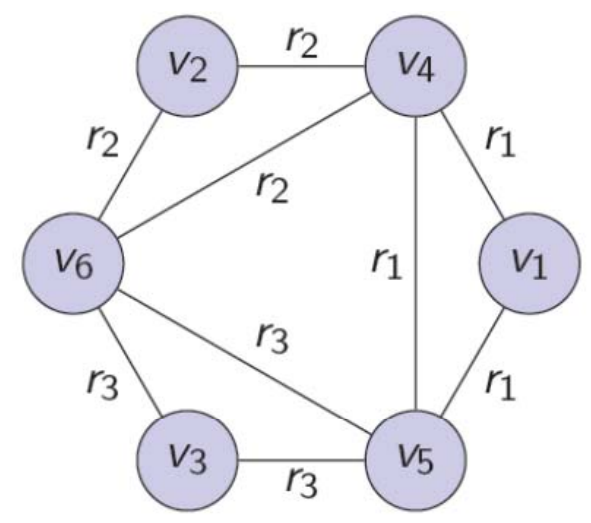

Figure 3. Non-overlapping graph 


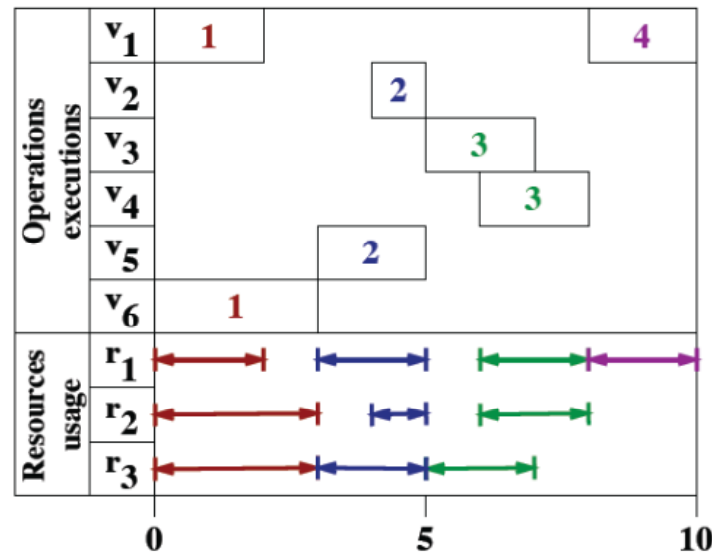

Figure 4. An illustrative solution schedule

In this paper we use the same approach by defining as non-overlapping operations those that occur in the same processing line, as well as any pair of shipping and processing operations. Shipping and processing are non-overlapping since loading and unloading of a tank cannot occur at the same time. The set $V$ is a collection of two types of operations: processing of an order in a finishing line and an unloading or shipping event. The first type is defined by a pair $(o, m) \in O \times M$; the second type of operation considers any shipping event $s \in S$. In set notation: $V=\{(o, m),(s): o \in O, m \in M, s \in S\}$. The cliques that appear in equations (MOS1) and (MOS2) are clique $_{m}(V)=\{(o, m),(s): o \in O, s \in S\}, \forall m \in M$. These cliques are result of defining that the processing of two orders on the same finishing line, and that loading and unloading of a tank must not overlap. As an example, consider the small system described in Table 2, where three processing orders have to be scheduled in two finishing lines. The finished products are stored in three tanks from which there will be shipped once to the customer. 
Table 2. Small illustrative example

\begin{tabular}{lccc} 
Processing orders & $o_{1}$ & $o_{2}$ & $o_{3}$ \\
Product in order $i$ & $A$ & $B$ & $C$ \\
Finishing lines & $m_{1}$ & $m_{2}$ & \\
Storage tanks & $T_{1}$ & $T_{2}$ & $T_{3}$ \\
Shipping events & $s_{1}$ & & \\
\hline
\end{tabular}

Table 3. Definition of operations in small illustrative Example

$\begin{array}{lllllll}\text { Processing operations } & \left(o_{1}, m_{1}\right) & \left(o_{2}, m_{1}\right) & \left(o_{3}, m_{1}\right) & \left(o_{1}, m_{2}\right) & \left(o_{2}, m_{2}\right) & \left(o_{3}, m_{2}\right)\end{array}$

Shipping operations $\quad s_{1}$

Figure 5 shows the non-overlapping graph that corresponds to the operations in Table 3.

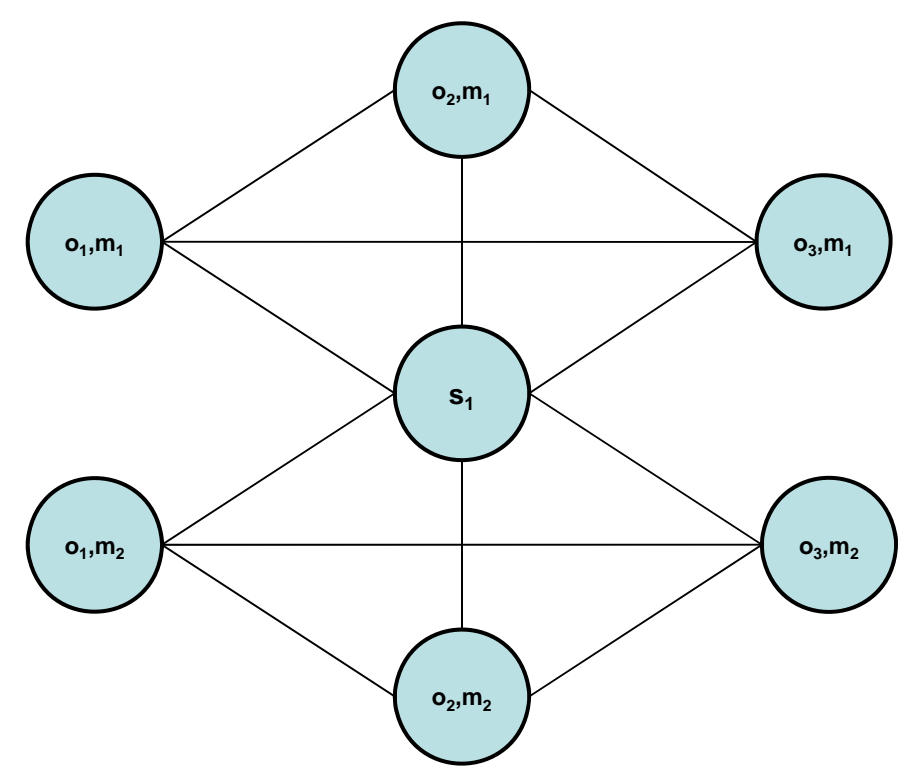

Fig. 5 Non-overlapping graph in small illustrative example

There are two cliques in the graph of Figure 5:

$$
\text { clique }_{m_{1}}=\left\{\left(o_{1}, m_{1}\right),\left(o_{2}, m_{1}\right),\left(o_{3}, m_{1}\right),\left(s_{1}\right)\right\} \text {, }
$$

and

clique $_{m_{2}}=\left\{\left(o_{1}, m_{2}\right),\left(o_{2}, m_{2}\right),\left(o_{3}, m_{2}\right),\left(s_{1}\right)\right\}$. 
Recalling that the set of slots is $L$, the non-overlapping constraint (MOS1) in the small illustrative example is written below:

$$
\sum_{v \in V^{*}} Z_{v, \ell} \leq 1 \quad \ell \in L, V^{*} \in \text { clique }_{m} \forall m \in M,
$$

or alternatively, if variable $Z_{v, \ell}$ is disaggregated into a variable for assigning processing operations to slots $w o_{o, m, \ell}$ and another for assigning shipping events to slots $w s_{s, \ell}$, $\sum_{o \in O} w o_{o, m, \ell}+\sum_{s \in S} w s_{s, \ell} \leq 1 \quad \ell \in L, m \in M$.

The latter form has the advantage that the cliques do not have to be explicitly defined, and that the constraint is described in terms of the naturally occurring sets $L$ and $M$. This is the form that we use in equations (4) and (5) of the mathematical model. Finally, a feasible schedule for this illustrative case using 3 priority time slots is found in Figure 6.

(a) Gantt Chart

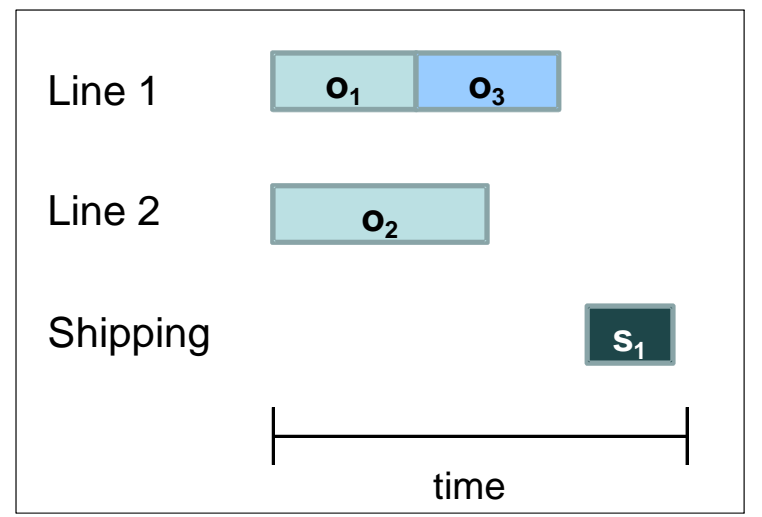

(b) Assignment of slots

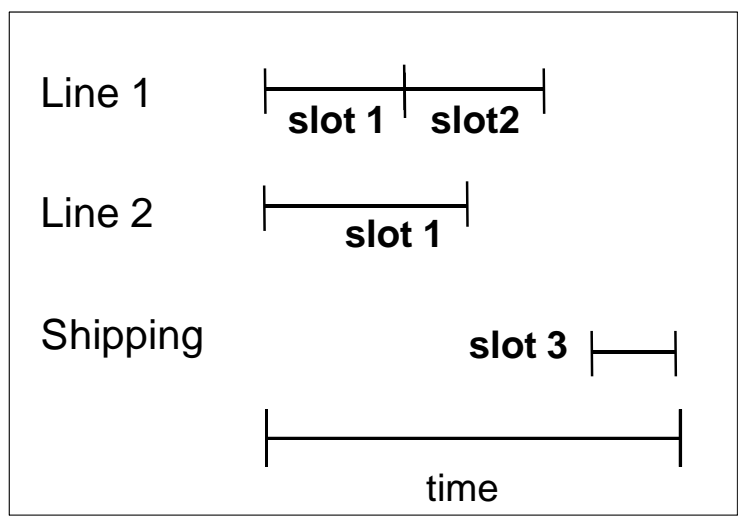

Fig. 6 A feasible schedule for the illustrative example

Sets/Index
$J / j$
Products
$K / k$
Tanks
$M / m$
Finishing lines
$L / \ell$
Time slots
$\mathrm{O} / \mathrm{O}$
Production orders 


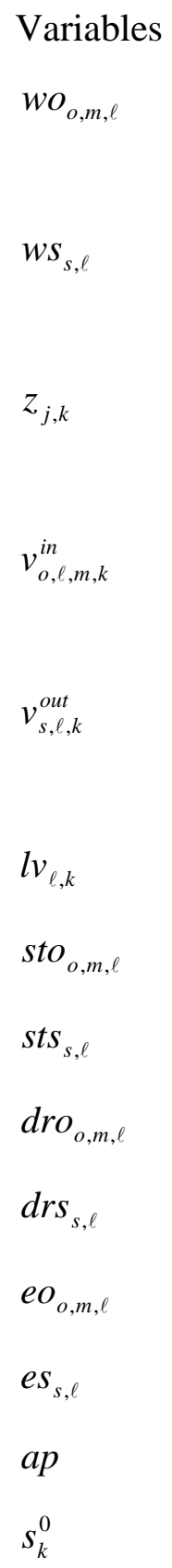




$\begin{array}{ll}d_{o, j} & \text { amount of finished product } j \text { requested by processing } \\ & \text { order } o \\ \delta_{o, j} & 1 \text { if product } j \text { is requested in processing order } o ; 0 \\ & \text { otherwise } \\ r d_{o} & \text { release date of processing order } o \\ c m p_{j, k} & 1 \text { if physical and chemical properties of product } j \text { are } \\ & \text { compatible with the operating conditions of tank } k ; 0 \\ & \text { otherwise } \\ & 1 \text { if any product can be transferred between line } \ell \text { and tank } \\ & k ; 0 \text { otherwise }\end{array}$

$m \times k_{j}$ maximum number of tanks that can be assigned to product $j$ $m n k_{j}$ minimum number of tanks that must be assigned to product $j$

rate $_{j, m}$ rate of production of product $j$ in line $m$ $s h_{s, j}$ shipping rate of product $j$ in shipping event $s$ shd $_{\mathrm{s}}$ stime $_{s}$

$\operatorname{shipwin}_{s}$ maximum duration of shipping event $s$ time when a shipping event $s$ is allowed to start; the maximum shipping event frequency is determined a priori, according to logistics of modes of transportation time window during which unloading of tank into modes transportation is allowed; the length of this shipping window is predetermined by the logistics of the process system

$\omega_{o}$ Weighting parameter of order $o$ according to its importance in the production schedule 


\section{Objective function}

The objective is to minimize the unallocated product. This objective is equivalent to maximizing the amount of finished product that is allocated to the storage tanks during the time horizon. The parameter $\omega_{o}$ is included to give different weights to different production orders. For instance, if an order is urgent it could have a large value of $\omega_{o}$. For all the results in this paper we set $\omega_{o}=1, \forall o \in O$.

$$
a p=\sum_{o \in O} \sum_{\ell \in L} \sum_{m \in M} \sum_{k \in K} \omega_{o} v_{o, \ell, m, k}^{\text {in }}
$$

\section{Scheduling constraints}

Equations (1a) and (1b) establish that the start time for the processing of order $o$ in line $m$ at slot $\ell$ is zero if the order is not assigned to that line during that slot, or otherwise it must be sometime after the release date and before the end of the horizon. Equation (1c) is a constraint on the frequency of shipping events. This equation fixes the start of each event $s$ at slot $\ell$ to either zero or the predetermined shipping time stime $e_{s}$. In equation (1d) the duration of the processing operation of order $o$ in line $m$ can only be non-zero if has to been assigned to slot $\ell$. The duration of a shipping event is either zero or less than the predetermined maximum duration of shipping events, as established by equation (1e). Equations (1f) and (1g) set the ending time of any slot to either zero if it is not assigned to processing or shipping, or to less than the operating horizon. Equations (2a) and (2b) establish that the ending time of any slot is equal to its starting time plus its duration.

$$
\begin{array}{ll}
s t o_{o, \ell, m} \leq w o_{o, \ell, m} H & o \in O, \ell \in L, m \in M \\
\text { sto }_{o, \ell, m} \geq w o_{o, \ell, m} r d_{o} & o \in O, \ell \in L, m \in M \\
s t s_{s, \ell}=w s_{s, \ell} \text { stime }_{s} & s \in S, \ell \in L \\
d r o_{o, \ell, m} \leq w o_{o, \ell, m} H & o \in O, \ell \in L, m \in M \\
d r s_{s, \ell} \leq w s_{s, \ell} \text { hhipwin }_{s} & s \in S, \ell \in L \\
e o_{o, \ell, m} \leq w o_{o, \ell, m} H & o \in O, \ell \in L, m \in M \\
e s_{s, \ell} \leq w s_{s, \ell} H & s \in S, \ell \in L
\end{array}
$$




$$
\begin{array}{ll}
e o_{o, \ell, m}=s t o_{o, \ell, m}+d r o_{o, \ell, m} & o \in O, \ell \in L, m \in M \\
e s_{s, \ell}=s t s_{s, \ell}+d r s_{s, \ell} & s \in S, \ell \in L
\end{array}
$$

It should be noted that the sum of all processing durations $d r o_{o, \ell, m}$ for each order are bounded by the sum of the product demands $d o_{o, j}$ as will be specified later in constraint (6).

By constraint (3a) an operation can be assigned at most once to any slot or finishing line; by constraint (3b) each shipping event only takes place once.

$$
\begin{array}{ll}
\sum_{\ell \in L} \sum_{m \in M} w o_{o, \ell, m} \leq 1 & o \in O \\
\sum_{\ell \in L} w S_{s, \ell} \leq 1 & s \in S
\end{array}
$$

Constraint (4) enforces that no overlapping operations are assigned to the same slot. It was described in more detail in the previous sections.

$$
\sum_{o \in O} w o_{o, \ell, m}+\sum_{s \in S} w s_{s, \ell} \leq 1 \quad \ell \in L, m \in M
$$

According to the idea of priority slots (Mouret et al., 2009), slot $\ell_{1}$ in equation (5) has a higher priority than slot $\ell_{2}$ in the sense that it takes place before or at the same time as slot $\ell_{2}$. If two non-overlapping operations, such as processing on the same finishing line or a shipping event and a processing operation, are assigned to $\ell_{1}$ and $\ell_{2}$, then $\ell_{2}$ must start after the end of $\ell_{1}$. Equation (5) enforces this constraint. The duration of the intermediate slots $\ell: \ell_{1}<\ell<\ell_{2}$ is considered in order to strengthen this constraint (Mouret et al., 2011).

$$
\begin{aligned}
& \sum_{o \in O} e o_{o, \ell_{1}, m}+\sum_{s \in S} e s_{s, \ell_{1}}+\sum_{\substack{\ell \in L \\
\ell_{1}<\ell<\ell_{2}}} d r o_{o, \ell, m}+\sum_{\substack{\ell \in L \\
\ell_{1}<\ell<\ell_{2}}} d r s_{s, \ell, m} \leq \\
& \sum_{o \in O} s t o_{o, \ell_{2}, m}+\sum_{s \in S} s t s_{s, \ell_{2}}+H\left(1-w o_{o, \ell_{2}, m}-w s_{s, \ell_{2}}\right)
\end{aligned} \quad \ell_{1}, \ell_{2}, \in L, \ell_{1}<\ell_{2}, m \in M
$$




\section{Tank transfer constraint}

Equations (6) and (7) allow the transfer of a finished product to storage tank $k$ from line $m$ during slot $\ell$, only if an order corresponding to that product is being processed, and if the tank is assigned to this product (i.e. $z_{j, k}=1$ ). In both constraints $\sum_{j \in J} d_{o, j}$ is a valid upper bound. Constraint (8) allows transfer between a line and a tank only if there is feasible connection (e.g., a pipe) between them. Constraint (9) sets the maximum rate of transfer to tanks equal to the rate of processing at the finishing lines. Equation (10) sets the maximum amount that can be unloaded from a tank for a shipping event in a given slot. The maximum rate of unloading is set by constraint (11)

$$
\begin{array}{ll}
\sum_{k \in K} v_{o, \ell, m, k}^{\text {in }} \leq w o_{o, \ell, m} \sum_{j \in J} d_{o, j} & o \in O, \ell \in L, m \in M \\
v_{o, \ell, m, k}^{\text {in }} \leq \sum_{j \in J}\left(z_{j, k} d_{o, j}\right) & o \in O, \ell \in L, m \in M, k \in K \\
v_{o, \ell, m, k}^{\text {in }} \leq t l c_{k, m} \sum_{j \in J} d_{o, j} & o \in O, \ell \in L, m \in M, k \in K \\
v_{o, \ell, m, k}^{\text {in }} \leq \sum_{j \in J}\left(\delta_{o, j} \text { rate }_{j, m} d r o_{o, \ell, m}\right) & o \in O, \ell \in L, m \in M, k \in K \\
v_{s, \ell, k}^{\text {out }} \leq w s_{s, \ell} v_{k} & s \in S, \ell \in L, k \in K \\
v_{s, \ell, k}^{\text {out }} \leq s h_{s, j} d r s_{s, \ell} & s \in S, \ell \in L, k \in K
\end{array}
$$

\section{Material balance in storage tanks}

The concept of priority slots $\ell_{1}, \ell_{2}$ where, $\ell_{1}<\ell_{2} \Rightarrow e t_{\ell_{1}} \leq s t_{\hat{\ell}_{2}}$, allows the material balance at each tank to be calculated as in constraint (12).

$$
l v_{\ell, k}=\sum_{m \in M o \in O} \sum_{\ell_{1}: \ell_{1} \leq \ell} v_{o, \ell_{1}, m, k}^{\text {in }}-\sum_{s \in S} \sum_{\ell_{1}: \ell_{1} \leq \ell} v_{s, \ell_{1}, k}^{\text {out }} \quad \ell \in L, k \in K
$$

Constraint (13) limits the level of inventory to the capacity of tank $k$,

$$
l v_{\ell, k} \leq v_{k} \quad \ell \in L, k \in K
$$




\section{Tank assignment constraints}

Equation (14) enforces the condition that at most one product can be assigned to each dedicated tank. Constraint (15) establishes the minimum and maximum number of tanks to which a product can be assigned. Constraint (16) allows an assignment only if the chemical and physical properties of a product are compatible with the operating conditions of a tank.

$$
\begin{array}{ll}
\sum_{j} z_{j, k} \leq 1 & k \in K \\
m n k_{j} \leq \sum_{k} z_{j, k} \leq m x k_{j} & j \in J \\
z_{j, k} \leq c m p_{j, k} & j \in J, k \in K
\end{array}
$$

Variable domain specifications

$$
\begin{array}{ll}
w o_{o, m, \ell} \in\{0,1\} & o \in O, \ell \in L, m \in M \\
w s_{s, \ell} \in\{0,1\} & s \in S, \ell \in L \\
z_{j, k} \in\{0,1\} & j \in J, k \in K \\
v_{o, \ell, m, k}^{\text {in }}, v_{s, \ell, k}^{\text {out }} \in \mathfrak{R}_{+} & o \in O, \ell \in L, m \in M, k \in K, s \in S \\
s t o_{o, \ell, m}, d r o_{o, \ell, m}, e o_{o, \ell, m} \in \Re_{+} & o \in O, \ell \in L, m \in M \\
s t s_{s, \ell}, d r s_{s, \ell}, e s_{s, \ell} \in \Re_{+} & s \in S, \ell \in L \\
l v_{\ell, k} \in \Re_{+} & \ell \in L, k \in K \\
a p \in \Re_{+} &
\end{array}
$$

\section{Example 1}

In this example we wish to determine the optimal tank assignment and optimal schedule for a system of five tanks and two finishing lines where three products are processed. We have a set of production orders that arrive during an interval of two weeks. This set of production orders is representative of the frequency of orders and the quantity ordered during long term operation of the system. Thus, it can be used for optimally assigning tanks to products. Tables $4-6$ contain the data required for the optimization model. In 
this example we consider that all products can be stored in all tanks, and that there is a feasible connection between every line and every tank.

Table 4. Production orders in Example 1

\begin{tabular}{|c|c|c|c|}
\hline Order & Product & $\begin{array}{c}\text { Quantity } \\
\text { [ton/hr] }\end{array}$ & $\begin{array}{c}\text { Release date } \\
{[\mathrm{hr}]}\end{array}$ \\
\hline 1 & $\mathrm{~A}$ & 105 & 0 \\
\hline 2 & $\mathrm{C}$ & 69 & 0 \\
\hline 3 & $\mathrm{C}$ & 35 & 48 \\
\hline 4 & $\mathrm{~B}$ & 98 & 72 \\
\hline 5 & $\mathrm{~A}$ & 110 & 96 \\
\hline 6 & $\mathrm{~B}$ & 56 & 168 \\
\hline 7 & $\mathrm{C}$ & 102 & 216 \\
\hline 8 & $\mathrm{~B}$ & 90 & 264 \\
\hline
\end{tabular}

Table 5. Production rate and tank capacities in Example 1

\begin{tabular}{|c|c|c|c|c|c|}
\multicolumn{2}{|c|}{ Production rate [ton/hr] } & & Tank & Capacity [ton] \\
\hline & Line 1 & Line 2 & & & \\
\hline A & 0.95 & 0.89 & & 1 & 90 \\
\hline B & 1.09 & 1.15 & & 2 & 120 \\
\hline C & 0.92 & 0.82 & & 3 & 85 \\
\hline & & & 4 & 110 \\
\hline & & & 5 & 70 \\
\hline
\end{tabular}

Table 6. Interval between shipping, duration of unloading, and unloading rate

\begin{tabular}{|c|c|c|c|}
\hline Tank & $\begin{array}{c}\text { Interval } \\
{[\mathrm{hr}]}\end{array}$ & $\begin{array}{c}\text { Duration } \\
{[\mathrm{hr}]}\end{array}$ & $\begin{array}{c}\text { Transfer rate } \\
{[\text { ton/hr] }}\end{array}$ \\
\hline 1 & 24 & 6 & 12.07 \\
\hline 2 & 24 & 6 & 13.86 \\
\hline 3 & 24 & 6 & 12.01 \\
\hline 4 & 24 & 6 & 12.31 \\
\hline 5 & 24 & 6 & 11.83 \\
\hline
\end{tabular}

The TFOP formulation described in the mathematical model section is used to solve Example 1. After some pre-analysis, 6 priority time slots are postulated. The main idea is that we need 4 slots to process the 8 orders in the 2 production lines, and, given the rates of unloading of tanks, about 2 or 3 slots for shipping operations. Computational results indicate that 6 priority slots yield the same solution as 7 but require less computational time. The resulting Mixed-Integer Linear Programming (MILP) model has 195 binary 
variables, 1,471 continuous variables, and 3,358 constraints. It was implemented in GAMS version 23.6 for Windows and solved using CPLEX 12.2 with an Intel Core i7 CPU at $2.93 \mathrm{GHz}$, and $4.00 \mathrm{~GB}$ of RAM. A solution within $0.2 \%$ of the optimum was found in 60 CPU seconds. The results are summarized in Table 7, and Figures 7 - 8. As seen in Table 7, 1.4 ton of product B cannot be allocated.

Table 7. Allocated product for Example 1

\begin{tabular}{|c|c|c|}
\hline Product & $\begin{array}{c}\text { Ordered amount } \\
\text { [ton] }\end{array}$ & $\begin{array}{c}\text { Allocated Quanity } \\
\text { [ton] }\end{array}$ \\
\hline A & 215 & 215 \\
\hline B & 244 & 242.6 \\
\hline C & 206 & 206 \\
\hline & & \\
\hline Total & 665 & 663.6 \\
\hline
\end{tabular}

Figure 7 shows the optimal product-tank assignment. The total production volume specified by the production orders of Product B is the highest, and it is assigned the largest total storage tank capacity. Product A has a larger production target than product $\mathrm{C}$, but product $\mathrm{A}$ is allotted less storage space. Figure 8 shows that the two production orders of product A are scheduled at the beginning and end of the time horizon, allowing for the complete unloading of the storage tank. This fact is observed in Figure 9(d). The Gantt chart in Figure 8 shows how the scheduling of non-overlapping operations, such as the processing operations in the same line, and processing and unloading operations are never scheduled in the same slot. From this figure we can also confirm that the ordering of the priority slots is maintained: every slot that has a lower numbering than a shipping slot ends before the shipping starts. 


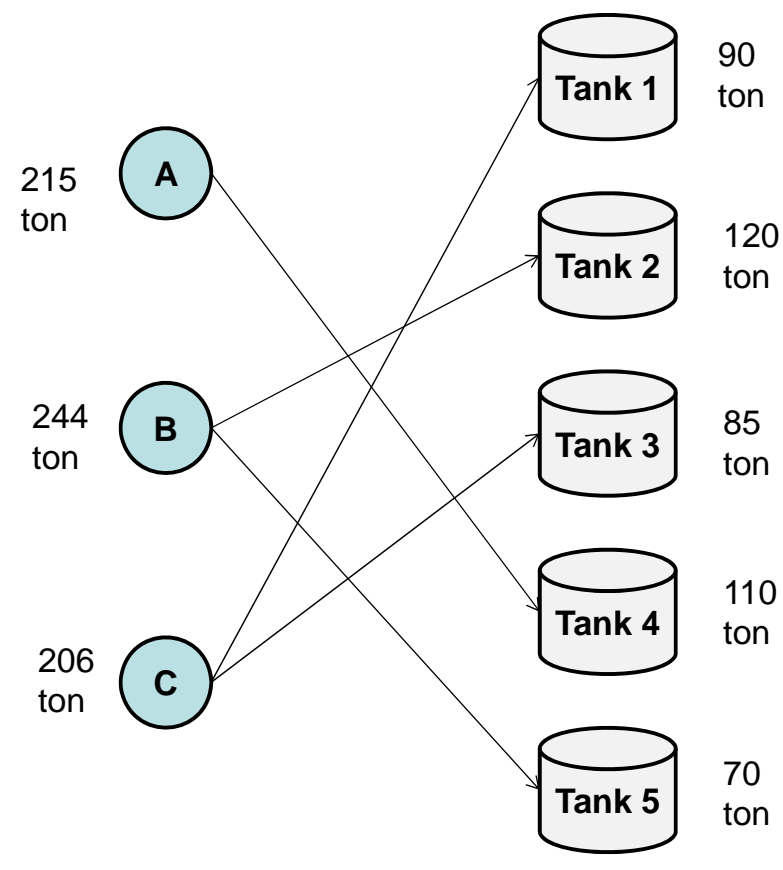

Fig 7. Optimal product-tank assignments in Example 1

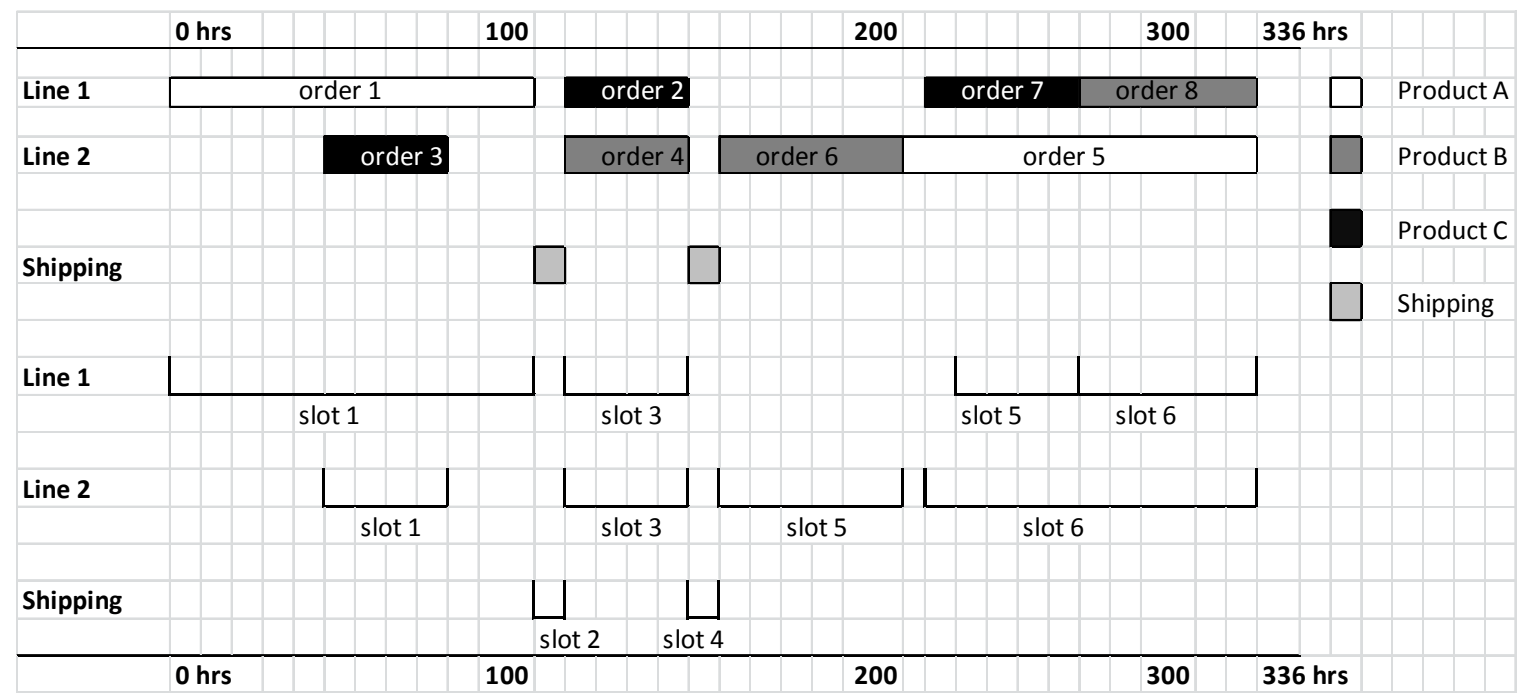

Fig. 8 Gantt chart of optimal schedule in Example 1

The plots of the tank levels in Figure 9 show a common pattern. Towards the end of the operating horizon all tanks except number 5 are completely full. This result is a combination of the objective function of the TFOP that requires a maximal amount of product going into the tanks, and the finite operating horizon. In real-life operations a shipping event would probably take place at the end of the time horizon. A cyclic 
schedule is an approach that eliminates this effect in case it is found to be an undesirable way of operating the system. We explore this alternative in Example 3.

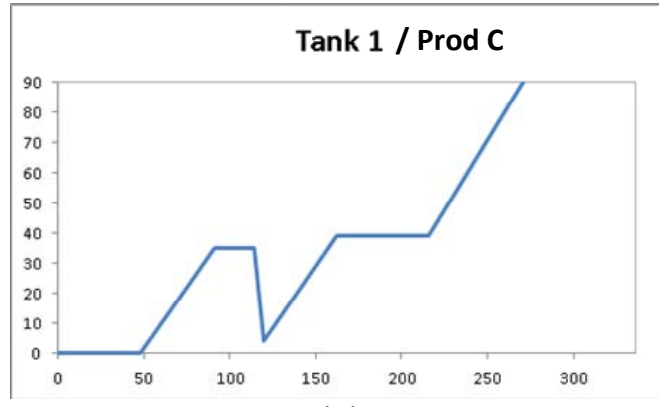

(a)

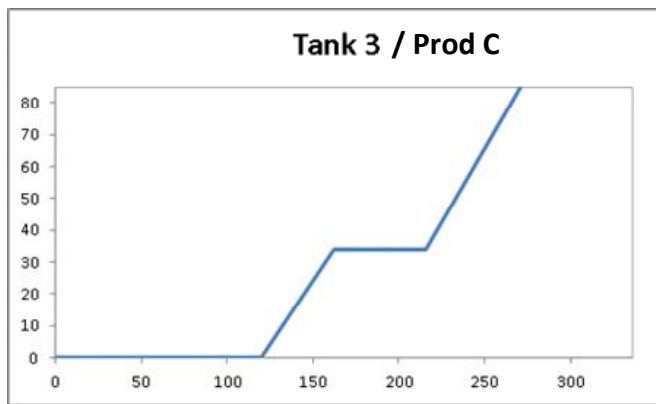

(c)

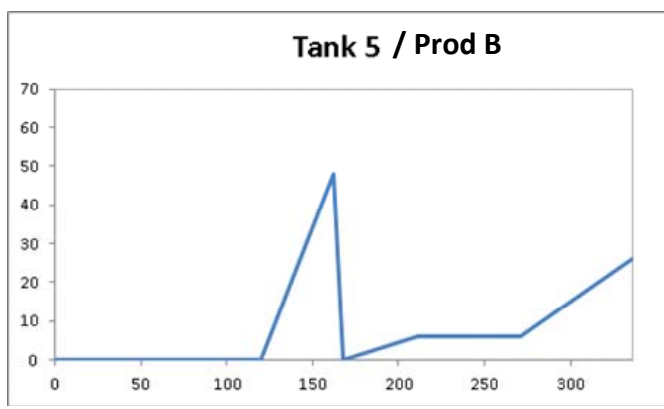

(e)

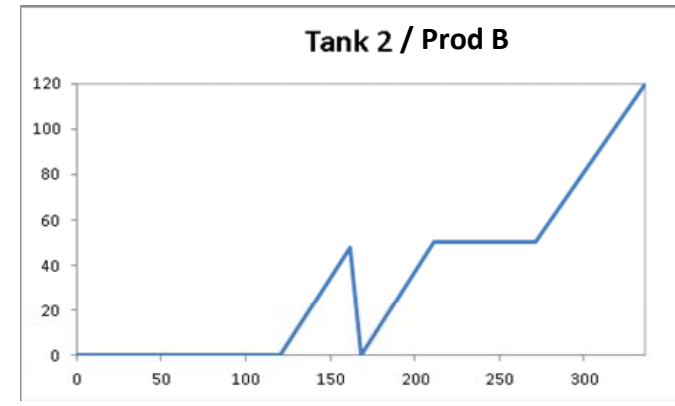

(b)

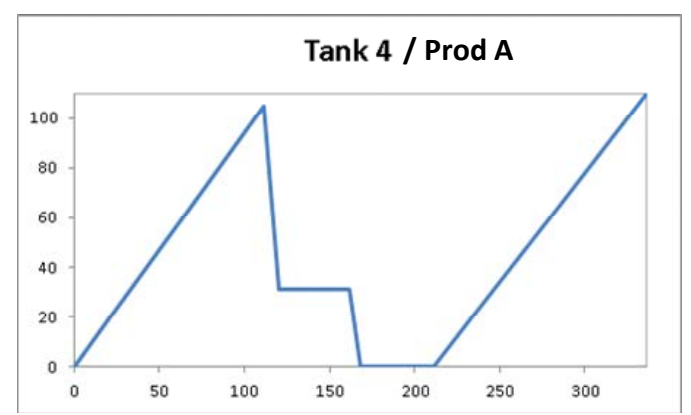

(d)

Fig. 9 Tank levels in the optimal solution to Example 1

\section{Example 2}

The purpose of this example is to highlight the advantage of solving the TFOP including scheduling of processing orders instead of considering a fixed processing schedule. We consider a system of two parallel finishing lines where 8 products are processed and fed into 10 storage tanks. The operating horizon is 672 hours (4 weeks). Tables $8-10$ contain the operating data of this system. 
Table 8. Production orders in Example 2

\begin{tabular}{|c|c|c|c|}
\hline Order & Product & $\begin{array}{c}\text { Quantity } \\
\text { [ton] }\end{array}$ & $\begin{array}{c}\text { Release date } \\
\text { [hr] }\end{array}$ \\
\hline 1 & P1 & 30 & 0 \\
\hline 2 & P1 & 40 & 0 \\
\hline 3 & P1 & 18 & 10 \\
\hline 4 & P2 & 10 & 14 \\
\hline 5 & P2 & 40 & 16 \\
\hline 6 & P2 & 20 & 18 \\
\hline 7 & P3 & 30 & 21 \\
\hline 8 & P3 & 20 & 124 \\
\hline 9 & P3 & 20 & 156 \\
\hline 10 & P4 & 30 & 198 \\
\hline 11 & P4 & 30 & 220 \\
\hline 12 & P4 & 10 & 272 \\
\hline 13 & P5 & 50 & 284 \\
\hline 14 & P5 & 30 & 316 \\
\hline 15 & P5 & 18 & 378 \\
\hline 16 & P6 & 25 & 380 \\
\hline 17 & P6 & 25 & 412 \\
\hline 18 & P7 & 30 & 544 \\
\hline 19 & P7 & 10 & 536 \\
\hline 20 & P8 & 25 & 558 \\
\hline 21 & P8 & 15 & 560 \\
\hline & & & \\
\hline
\end{tabular}

Table 9. Production rate and tank capacities in Example 2

\begin{tabular}{|l|l|l|l|l|l|}
\hline \multicolumn{3}{|c|}{ Production rate [ton/hr] } & & Tank & Capacity [ton] \\
\hline Line 1 & Line 2 & & & \\
\hline P1 & 1.29 & 1.29 & & T1 & 30 \\
\hline P2 & 1.07 & 1.07 & & T2 & 27 \\
\hline P3 & 1.07 & 1.07 & & T3 & 18 \\
\hline P4 & 1.07 & 1.07 & & T4 & 15 \\
\hline P5 & 1.64 & 1.64 & & T5 & 15 \\
\hline P6 & 0.55 & 0.55 & & T6 & 15 \\
\hline P7 & 0.71 & 0.71 & & T7 & 12 \\
\hline P8 & 0.71 & 0.71 & & T8 & 12 \\
\hline & & & T9 & 15 \\
\hline & & & & T10 & 39 \\
\hline
\end{tabular}


Table 10. Interval between shipping, duration of unloading, and unloading rate

\begin{tabular}{|c|c|c|c|}
\hline Tank & $\begin{array}{c}\text { Interval } \\
{[\mathrm{hr}]}\end{array}$ & $\begin{array}{c}\text { Duration } \\
{[\mathrm{hr}]}\end{array}$ & $\begin{array}{c}\text { Transfer rate } \\
\text { [ton/hr] }\end{array}$ \\
\hline 1 & 24 & 4 & 2.50 \\
\hline 2 & 24 & 4 & 2.25 \\
\hline 3 & 24 & 4 & 1.50 \\
\hline 4 & 24 & 4 & 1.25 \\
\hline 5 & 24 & 4 & 1.25 \\
\hline 6 & 24 & 4 & 1.25 \\
\hline 7 & 24 & 4 & 1.00 \\
\hline 8 & 24 & 4 & 1.00 \\
\hline 9 & 24 & 6 & 0.83 \\
\hline 10 & 24 & 6 & 2.17 \\
\hline
\end{tabular}

In the case where the schedule is fixed each production order starts exactly at the release date and lasts until it is completed or until a new order has to be processed. Every time that the tanks block the finishing lines, there is a quantity of unallocated product generated that is equal to the time the line was blocked multiplied by the processing rate of the line. The 21 production orders can in principle be processed in 11 priority slots by the two finishing lines, and we estimated 2 or 3 shipping events per week. After some computational experiments we specified 18 priority slots. The resulting MILP has 1,340 binary variables, 16,561 continuous variables, and 40,261 constraints. We solve it with the same hardware as in Example 1. However, instead of using the solver CPLEX as we did in Example 1, we use Gurobi 4.0. Computations with both solvers showed that for this example Gurobi found better solution in about an order of magnitude less time than CPLEX. Table 11 shows the best solutions and solution times found with fixed schedule and with optimal scheduling. Note that fixing the schedule actually makes the problem harder to solve, presumably because it becomes more constrained.

Table 11. Computational results of the TFOP with fixed and optimal scheduling

\begin{tabular}{ccc}
\hline & Fixed Scheduling & Optimal Scheduling \\
\hline Best Solution [ton] & 439 & 517 \\
Optimality Gap [\%] & 19.9 & 1.7 \\
Linear Relaxation [ton] & 526 & 526 \\
CPU [second] & 10,000 & 530 \\
\hline
\end{tabular}


The total quantity required by production orders is 526 tons. The best solution with fixed schedule (439 tons) after 10,000 CPU s corresponds to approximately 16\% unallocated product, while a solution of 517 tons involving less than $2 \%$ unallocated product is found when optimal scheduling is included in the TFOP. Table 8 shows that seven production orders are released between hour 0 and hour 21. A similar accumulation of orders occurs between hours 540 and 560 . When the schedule requires each order to be processed at its release date, as is the case with fixed schedule, some orders have to be cut short or missed all together.

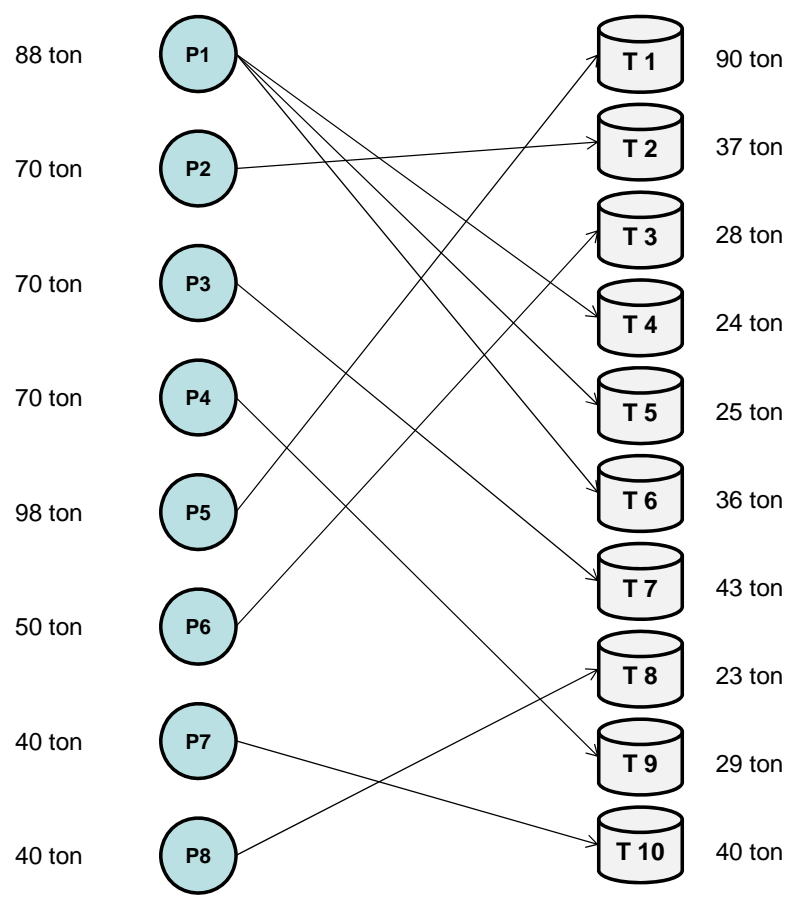

Fig. 10 Optimal tank assignments with fixed schedule 


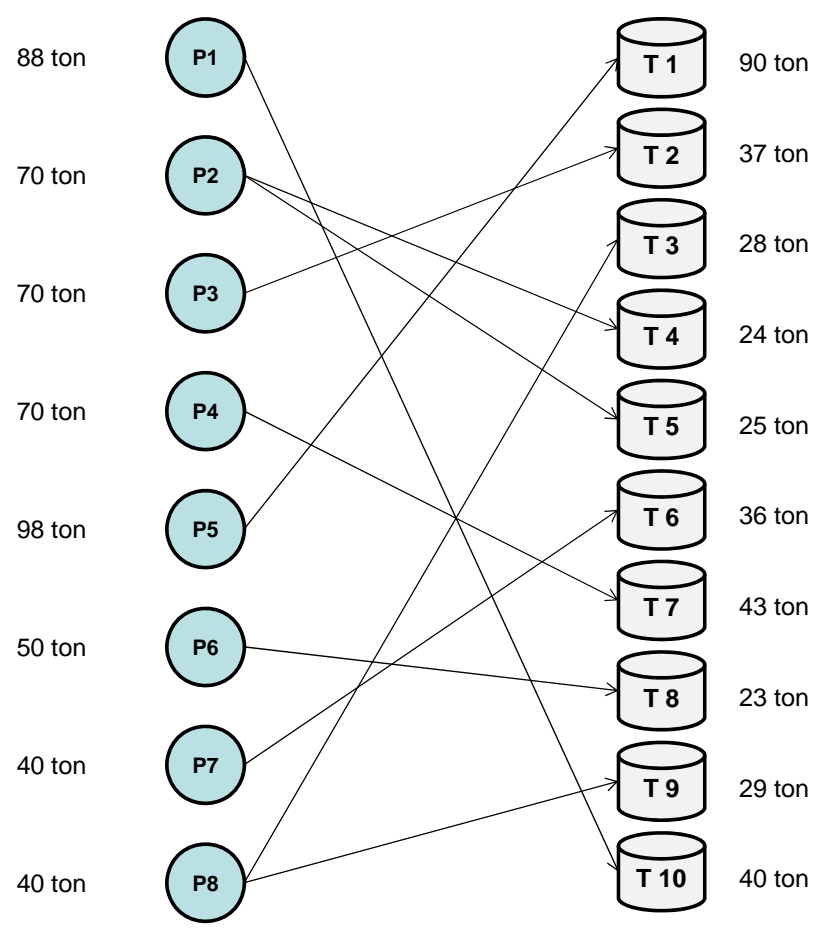

Fig. 11 Optimal tank assignments with optimal schedule

The main point in this example is that including the optimal scheduling in the TFOP has a significant effect on the quality of the solution found and on the computation times required. When scheduling is included, a solution within $2 \%$ of the optimum and involving almost no unallocated product can be found in 530 CPUs. In contrast, the best solution found after 10,000 CPUs for the problem with fixed scheduling involves significant amounts of unallocated product and an optimality gap of $~ 20 \%$.

\section{Example 3}

This example involves the same system as in Example 2 but with an extended time horizon of 6 weeks (1008 hrs.), which is longer than usual for scheduling problems. The reason is that we require a schedule that is representative of the medium to long-term operation of the plant in order to make the tank allocation decisions. An important difference from previous examples is that we consider a cyclic operating mode where the cycle time is 1008 hrs. A new variable, $s_{k}^{0}$, is introduced in the equation for the inventory balance, yielding equation (25).

$$
l v_{\ell, k}=s_{k}^{0}+\sum_{m \in M} \sum_{o \in O} \sum_{\ell_{1}: \ell_{1} \leq \ell} v_{o, \ell_{1}, m, k}^{\text {in }}-\sum_{s \in S} \sum_{\ell_{1}: \ell_{1} \leq \ell} v_{s, \ell_{1}, k}^{\text {out }} \quad \ell \in L, k \in K
$$


The following constraint to enforce similar initial and final inventory is added to the model:

$$
s_{k}^{0}-\varepsilon_{k} \leq v_{\ell, k} \leq s_{k}^{0}+\varepsilon_{k} \quad \ell=|L|, k \in K,
$$

where $\varepsilon_{k}$ is a small scalar introduced to constraint (26) in order to relax the strict equality between initial and final inventory in cyclic scheduling. Computations showed that this relaxation has a significant effect on the speed of convergence of the MILP solvers we used. For this case study, we set $\varepsilon_{k}$ to 2. For instance, increasing the value of $\varepsilon_{k}$ from 2 to 5 reduces the required CPU time to about one fourth. However, we considered a value of 2 the largest reasonable slack in equation (26).

To ensure the slot $\ell=|L|$ corresponds chronologically to the last slot, we enforce the following precedence constraint:

$$
\sum_{o \in O} e o_{o, \ell_{1}, m}+\sum_{s \in S} e s_{s, \ell_{1}} \leq \sum_{o \in O} s t o_{o, \ell_{2}, m}+\sum_{s \in S} s t s_{s, \ell_{2}} \quad \ell_{1} \in L, \ell_{2}=|L|, m \in M
$$

Table 12 contains data of the orders corresponding to the extra two weeks in Example 3. Selecting 24 priority slots, the resulting MILP has 2,500 binary variables, 31,691 continuous variables, and 77,212 constraints. 
Table 12. Processing orders in weeks 5 and 6 in Example 3

\begin{tabular}{|c|c|c|c|}
\hline Order & Product & $\begin{array}{c}\text { Quantity } \\
\text { [ton] }\end{array}$ & $\begin{array}{c}\text { Release date } \\
{[\mathrm{hr}]}\end{array}$ \\
\hline 1 & P1 & 30 & 0 \\
\hline 2 & P1 & 40 & 0 \\
\hline 3 & P1 & 18 & 10 \\
\hline 4 & P2 & 10 & 14 \\
\hline 5 & P2 & 40 & 16 \\
\hline 6 & P2 & 20 & 18 \\
\hline 7 & P3 & 30 & 21 \\
\hline 8 & P3 & 20 & 124 \\
\hline 9 & P3 & 20 & 156 \\
\hline 10 & P4 & 30 & 198 \\
\hline 11 & P4 & 30 & 220 \\
\hline 12 & P4 & 10 & 272 \\
\hline 13 & P5 & 50 & 284 \\
\hline 14 & P5 & 30 & 316 \\
\hline 15 & P5 & 18 & 378 \\
\hline 16 & P6 & 25 & 380 \\
\hline 17 & P6 & 25 & 412 \\
\hline 18 & P7 & 30 & 544 \\
\hline 19 & P7 & 10 & 536 \\
\hline 20 & P8 & 25 & 558 \\
\hline 21 & P8 & 15 & 560 \\
\hline & & & \\
\hline
\end{tabular}

Table 13 shows the best solution found after 7 hours of computations. The product tank assignment is shown in Figure 12, while the initial and final inventories are shown in Table 14.

Table 13. Computational results of the TFOP with cyclic scheduling

\begin{tabular}{lc}
\hline Best Solution [ton] & 769 \\
Unallocated product [\%] & 7.5 \\
Optimality Gap [\%] & 5.9 \\
CPU [second] & 24,538 \\
\hline
\end{tabular}




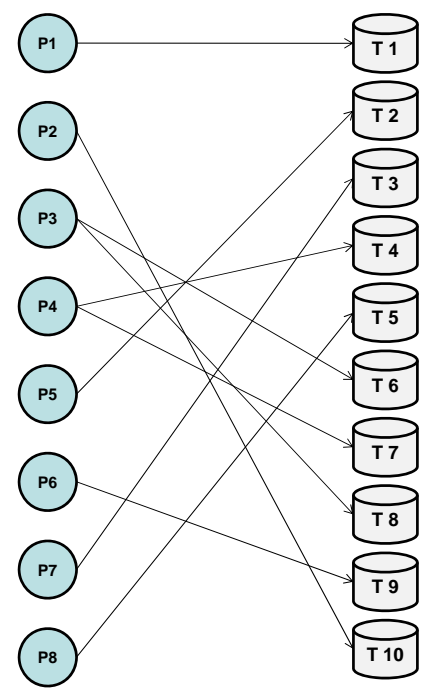

Figure 12 Tank assignments with cyclic schedule

Table 14. Initial and final inventory in storage tanks

\begin{tabular}{lccc}
\hline Tank & $\begin{array}{c}\text { Capacity } \\
\text { [ton] }\end{array}$ & $\begin{array}{c}\text { Initial inventory } \\
\text { [ton] }\end{array}$ & $\begin{array}{c}\text { Final inventory* } \\
\text { [ton] }\end{array}$ \\
\hline T1 & 90 & 0.0 & 2.0 \\
T2 & 37 & 19.0 & 21.0 \\
T3 & 28 & 0.0 & 2.0 \\
T4 & 24 & 8.8 & 10.8 \\
T5 & 25 & 0.0 & 2.0 \\
T6 & 36 & 12.0 & 14.0 \\
T7 & 27.6 & 29.6 \\
T8 & 43 & 13.0 & 15.0 \\
T9 & 23 & 13.3 & 15.3 \\
T10 & 29 & 38.0 & 40.0 \\
\hline
\end{tabular}

*Final inventory $=$ Initial inventory $+/-2$

After 7 hours of CPU time the optimality gap is still 6\%. We are interested in obtaining a lower bound on the amount of unallocated product (an upper bound to equation obj) that would be possible to achieve if storage tank space was not a limitation. Therefore, we solve a relaxation of Example 3 that involves unlimited storage capacity.

Remark Since we allow several lines to feed the same tank simultaneously, and we assume there is at least as many tanks as products, unlimited storage capacity turns the 
product-assignment into a meaningless decision variable. For this reason we a priori assign a product to a tank arbitrarily.

Table 15. Computational results of the TFOP with cyclic scheduling with unlimited tank sizes

\begin{tabular}{lc}
\hline Best Solution [ton] & 828 \\
Unallocated product [\%] & 0.4 \\
Optimality Gap [\%] & 0.4 \\
CPU [second] & 219 \\
\hline
\end{tabular}

Table 16. Storage requirements, initial, and final inventory in TFOP with unlimited tank sizes

\begin{tabular}{lccc}
\hline Product & $\begin{array}{c}\text { Maximum inventory } \\
\text { [ton] }\end{array}$ & $\begin{array}{c}\text { Initial inventory } \\
\text { [ton] }\end{array}$ & $\begin{array}{c}\text { Final inventory* } \\
\text { [ton] }\end{array}$ \\
\hline P1 & 76.0 & 46.0 & 48.0 \\
P2 & 98.0 & 58.0 & 60.0 \\
P3 & 50.0 & 0.0 & 2.0 \\
P4 & 65.1 & 25.1 & 27.1 \\
P5 & 50.0 & 0.0 & 2.0 \\
P6 & 50.0 & 0.0 & 0.0 \\
P7 & 40.0 & 0.0 & 0.0 \\
P8 & 25.0 & 3.0 & 5.0 \\
\hline
\end{tabular}

* Final inventory = Initial inventory $+/-2$.

Comparing Tables 14 and 16 we can see the storage requirement for the solution corresponding to 828 tons of allocated product (vs. 769 tons in the finite storage setting) requires more storage capacity than what is available in the tank farm. The total storage capacity required to meet the maximum inventory levels obtained from solving the problem assuming unlimited storage capacity is 454 tons, while the original problem with finite storage has a total capacity of 375 tons. This type of analysis could be used to evaluate capital investment decisions in tank farms. 


\section{Conclusions and future work}

We have presented a novel MILP formulation for the Tank Farm Operation Problem (TFOP) that integrates continuous production scheduling with storage resource allocation when the storage vessels are dedicated tanks. One of the examples in this paper shows the impact of including optimal scheduling in the TFOP, as opposed to assuming a fixed schedule. Even though the scheduling part of the problem corresponds to an efficient model for continuous-time scheduling based on the idea of Multi-operation Sequencing (Mouret et al., 2011), the scheduling horizon that can be contemplated within reasonable computational time is limited to a few weeks. For this reason a representative set of production orders and release dates has to be chosen in order to obtain an efficient product-assignment for medium to long-term operation. Alternatively, a cyclic schedule can be assumed as we did in Example 3.

We envision our MILP formulation combined with Discrete Event Simulation like the one in Sharda and Vazquez (2009) as part of a comprehensive decision support system. Consequently, testing and integration with a simulation tool is a natural next step. In this way the MILP capabilities of rigorous search among alternative could be combined with the capability for representing complex operational issues of DES.

\section{Acknowledgment}

The authors acknowledge Naoko Akiya and Bikram Sharda for their contributions to defining this problem. The authors are also grateful for the financial support provided by the Dow Chemical Company.

\section{References}

Banks J., J.S. Carson II, B.L. Nelson, and D.M. Nicol (2005). Discrete event system simulations. $4^{\text {th }}$ ed. Prentice Hall International series in Industrial and Systems.

Cassandras C.G. (1993). Discrete Event Systems: Modeling and Performance Analysis. Irwin Publ., 1993.

Chen, E.J., M.L. Young, and P.L. Selikson (2002). A simulation study of logistic activities in a chemical plant. Simulation Modeling practice and Theory, 10, 235 - 245.

Chryssolouris G., N. Papakostas, and D. Mourtzis (2005). Refinery short-term scheduling with tank farm, inventory and distillation management: An integrated simulation based approach. European Journal of Operational Research, 166, 812 - 827. 
Erdirik-Dogan M. and I. E. Grossmann (2008). Simultaneous planning and scheduling of single-stage multiproduct continuous plants with parallel lines. Computers and Chemical Engineering, 32, 2664 2683.

Floudas C.A. and X. Lin (2004). Continuous-time versus discrete-time approaches for scheduling of chemical processes: a review. Computers and Chemical Engineering, 28, 2109 - 2129.

Furman, K.C., Z. Jia, and M.G. Ierapetritou (2007). A robust event-based continuous time formulation for tank transfer scheduling. Industrial and Engineering Chemistry Research, 46, 9126 - 9136.

Ha J., H. Chang, E. S. Lee, In. Lee, B.S. Lee, and G. Yi (2000). Intermediate storage tank operation strategies in the production scheduling of multi-product batch process. Computers and Chemical Engineering, 24, 1633 - 1640.

Hvattum, L.M., K. Fagerholt, V. A. Armentano (2009). Tank allocation problems in maritime bulk shipping. Computers and Operations Research, 36, 3051 - 3060.

Imagine That Inc. (2007). ExtendSim User Guide, Release 7.

Kondili E., C.C. Pantelides, and R.W.H. Sargent (1993). A general algorithm for short-term scheduling of batch operations-I. MILP formulation. Computers and Chemicals Engineering, 2, 211 - 227.

Kopanos, G.M., L. Puigjaner, C. T. Maravelias (2011). Production planning and scheduling of parallel continuous processes with product families. Industrial and Engineering Chemistry Research 50, 1369 1378.

Lima, R.M., I.E. Grossmann, and Y. Jiao (2011). Long-term scheduling of a single-unit multi-product continuous process to manufacture high performance glass. Computers and Chemical Engineering 35, $554-574$.

Mouret S., I.E. Grossmann, and P. Pestiaux (2009). A novel priority-based continuous-time formulation for crude-oil scheduling problems. Industrial and Engineering Chemistry Research, 48, 8515 - 8528.

Mouret S., I.E. Grossmann, and P. Pestiaux (2011).Time representations and mathematical models for process scheduling problems. Computers and Chemical Engineering, 35, 1038 - 1063.

Pantelides, C.C. (1994). Unified frameworks for process planning and scheduling. In Foundations of computer-aided process operations. New York: CACHE publications, pp. $253-274$.

Sharda B., and A. Vazquez (2009). Evaluating Capacity and Expansion Opportunities at Tank Farm: A Decision Support System using Discrete Event Simulation. Winter Simulation Conference 2009, 2218 2224.

Stewart M.D. and L.D. Trierwiler (2005). Simulating optimal tank farm design. PTQ magazine, Q2, 1 - 5.

Vecchietti, A.R., J. Montagna (1998). Alternatives in the optimal allocation of intermediate storage tanks in multiproduct batch plants. Computers and Chemical Engineering, 22(1), S801 - S804.

Zeng Q. and Z. Yang (2009). Integrating simulation and optimization to schedule loading operations in container terminals. Computers and Operations Research, 36, 1935 - 1944.

\section{Appendix A}

In this section we present the reasoning for choosing the MOS model over two alternative scheduling formulations in discrete and continuous time. We base the selection of the algorithm on the combinatorial complexity of the model measured by the number of binary variables required in each model.

A discrete time model (DTM) requires binary variable $w_{o, m, t_{d}}^{1}$ to indicate the production of order $o$ in line $m$ during time interval $t_{d}$. The use of this variable is illustrated in Figure A.1. The model is of order $O \times M \times T_{d}$. The model EDG by Erdirik-Dogan and 
Grossmann (2008) requires a binary variable $w_{o, m, \ell, t}^{2}$ for each order $o$, processing line $m$, time slot $\ell$, and time interval $t$. Each time period has a predetermined number of processing slots as shown in Figure A.2. This model has a number of binary variables in the order of $O \times M \times \sum_{t \in T_{c}} L_{t}$. Finally, the model MOS by Mouret et. al (2011) requires a binary variable $w_{o, m, \ell}^{3}$ for each order $o$, finishing line $m$, and processing slot $\ell$. The model is of size $O \times M \times L$ in terms of binary variables (Figure A.3). In this case the number of processing slots is also predetermined. It is important to note that the number of time intervals $T_{d}$ in the discrete model is not necessarily the same as $T_{c}$ in the continuous time model EDG of Erdirik-Dogan and Grossmann (2008). Likewise, the number of slots in each time period in this last model $L_{t}$ is not the same as the total number of slots $L$ in the continuous time model MOS by Mouret et. al (2011).

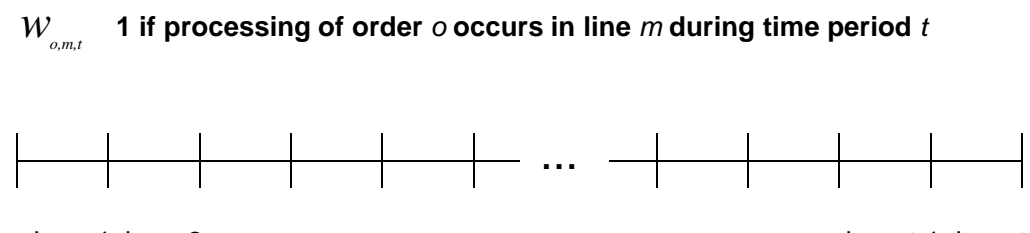

hour 1 hour 2

hour t-1 hour t

Line 1

\begin{tabular}{|l|l|l}
\hline order 1 & order 3 & \\
\hline
\end{tabular}

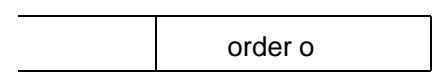

Line $\mathrm{m}$

order 2

\begin{tabular}{|l|l|}
\hline order $0-2$ & order $0-1$ \\
\hline
\end{tabular}

Fig. A.1 Discrete time representation 
$W_{o, m, \ell, t} 1$ if processing of order $o$ occurs in line slot $\ell$ of line $m$ during time period $t$

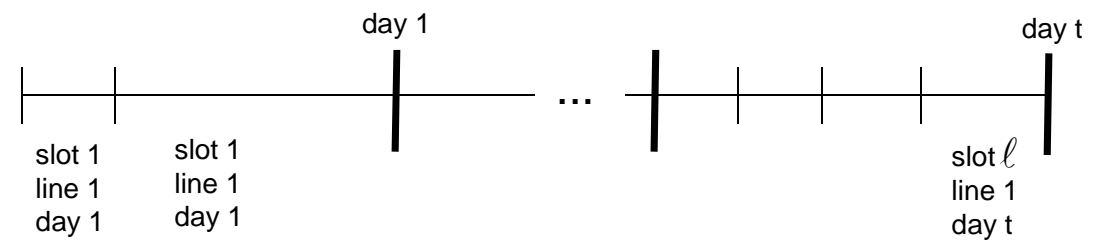

Line 1

\begin{tabular}{|l|l|l}
\hline order 1 & order 3 & \\
\hline
\end{tabular}
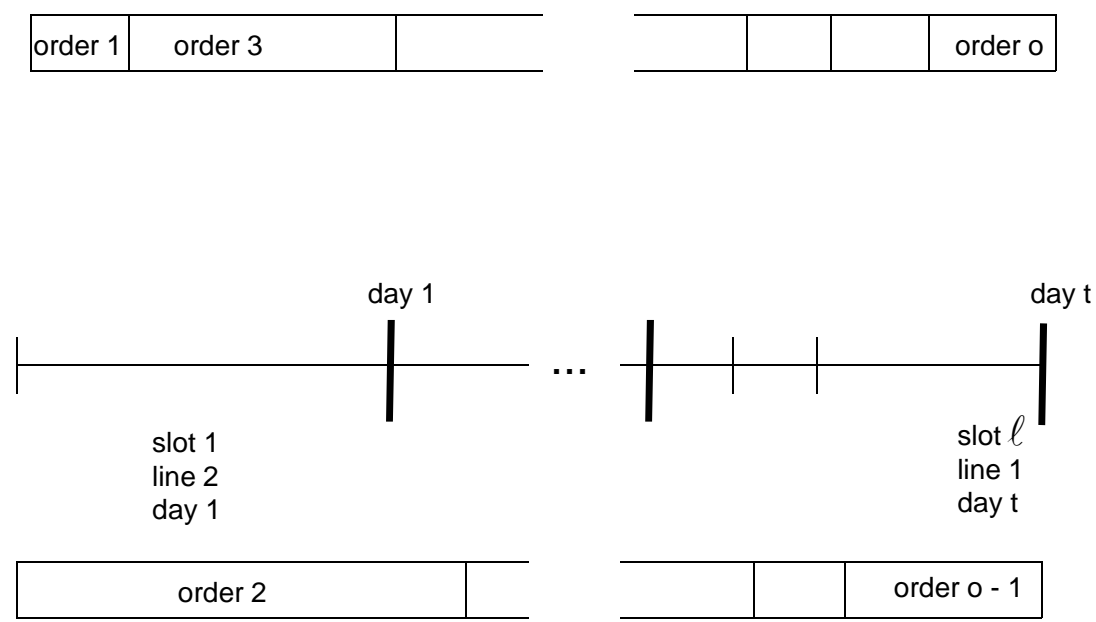

Fig. A.2 Continuous time representation in the model EDG

$W_{o, m, \ell, t} 1$ if processing of order $o$ occurs in line slot $\ell$ of line $m$ during time period $t$

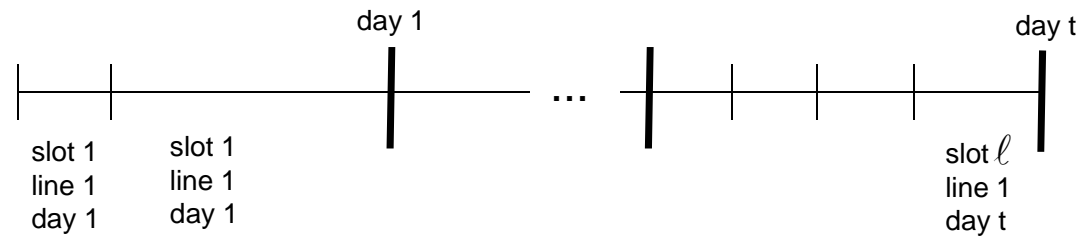

Line 1
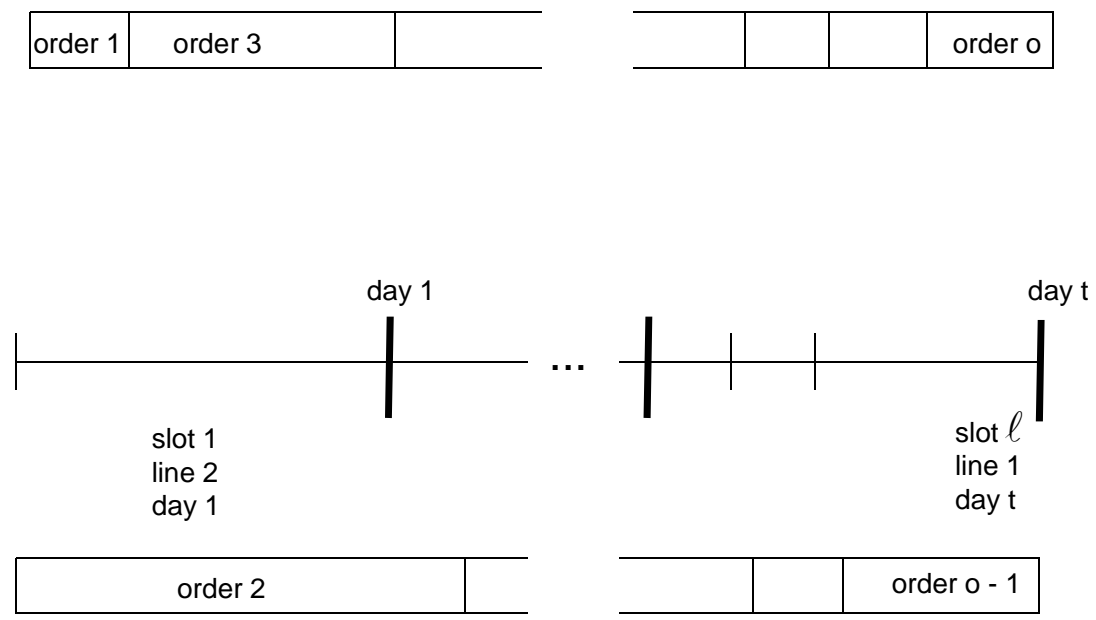

Fig. A.3 Continuous time representation in the MOS model 
For a given problem with a certain number of orders $O$ to be processed in a set $L$ of finishing lines, we can estimate the order of the number of binary variables required in each model. To do so, we exploit certain operational conditions: (i) each order cannot be split into two processing steps; it has to be completed once it is started, and (ii) shipping or unloading of the tanks occurs regularly with intervals much smaller than the time horizon, but larger than the required interval in discrete time optimization. The first operational condition above allows us to postulate that the maximum number of slots in the MOS model by Mouret et. al (2011) is at most equal to the number of orders. In the model EDG by Erdirik-Dogan and Grossmann (2008) we should postulate a number of processing slots for each time period equal to all the processing orders released before the start time of the time period. Also, from the operational constraint (ii), we have to impose that the time intervals $t_{c} \in T_{c}$ in the model EDG by Erdirik-Dogan and Grossmann (2008) coincide with the shipping intervals in order to keep track of inventory levels in storage tanks. Then, form operational characteristic (ii), we can establish that horizon $>>\Delta t_{c}>\Delta t_{d}$ and $T_{d}>T_{c}$.

The number of binary variables in each model is:

- $\quad D T M=O \times M \times T_{d}$

- $E D G=O \times M \times \sum_{t \in T_{d}} L_{t}=O \times M \times \overline{L_{t}} \times T_{c}$, where $\overline{L_{t}}$ is the average number of slots in each time period.

- $M O S=O \times M \times L=O \times M \times O$.

Since the number of orders and lines is the same in each model, it is enough to compare the following quantities: $T_{d}, \overline{L_{t}} \times T_{c}$, and $O$.

To give a concrete reference to these magnitudes we specify that DTM uses intervals of 4 hours. This means that if the average time between releasing of orders is more than 4 hours then there will be more time intervals in the discrete time model than the total number of orders. Thus $T_{d}>O$. 
For the EDG model, we consider that each time interval is one day (unloading of tanks occurs once a day). We also specify that each week a fixed number of new processing orders are released. This specification means that a plot of orders released vs. time will be linear, starting at 0 orders at time 0 , and ending with $O$ orders at the end of the time horizon. Therefore, the average number of orders released per time interval is equal to $\frac{O}{2}$. The average number of slots in each time interval in the EDG model can be set equal to the average number of orders, that is $\overline{L_{t}}=\frac{O}{2}$. Then, if the scheduling horizon is more than two days $\overline{L_{t}} \times T_{c}=\frac{O}{2} \times T_{c}>O$.

Under this assumptions that resemble real operating conditions:

$D T M=O \times M \times T_{d}>M O S=O \times M \times L$

and

$$
E D G=O \times M \times \overline{L_{t}} \times T_{c}>M O S=O \times M \times L
$$

We recognize that the number of binary variables is not always a reliable indicator of the computational time required for a solving a scheduling model. The tightness of the linear relaxation and the model structure are also important. However, we think that the number of binary variables is a good indicator for selecting the scheduling model. 